\title{
Breakdown of Kolmogorov scaling in models of cluster aggregation with deposition
}

\author{
Colm Connaughton, ${ }^{1}$ R. Rajesh, ${ }^{2}$ and Oleg Zaboronski ${ }^{3}$ \\ ${ }^{1}$ Laboratoire de Physique Statistique de l'ENS, 24 rue Lhomond - 75231 Paris cedex 05, France \\ ${ }^{2}$ Martin Fisher School of Physics, Brandeis University, Mailstop 057, Waltham, MA 02454-9110, USA \\ ${ }^{3}$ Mathematics Institute, University of Warwick, Gibbet Hill Road, Coventry CV4 7AL, UK
}

(Dated: November 20, 2018)

\begin{abstract}
The steady state of the model of cluster aggregation with deposition is characterized by a constant flux of mass directed from small masses towards large masses. It can therefore be studied using phenomenological theories of turbulence, such as Kolmogorov's 1941 theory. On the other hand, the large scale behavior of the aggregation model in dimensions lower than or equal to two is governed by a perturbative fixed point of the renormalization group flow, which enables an analytic study of the scaling properties of correlation functions in the steady state. In this paper, we show that the correlation functions have multifractal scaling, which violates linear Kolmogorov scaling. The analytical results are verified by Monte Carlo simulations.

PACS numbers: 05.10.Cc,47.27.Gs,05.70.Ln,68.43.Jk
\end{abstract}

Understanding Navier-Stokes turbulence is a big challenge of modern theoretical physics. It is a nonlinear system far from equilibrium with no obvious small parameters that would allow a perturbative treatment. It would therefore be instructive to study simpler nonequilibrium models which possess the qualitative features of turbulent systems, yet are analytically tractable. Such an approach has proved to be fruitful, especially during the past decade. The study of Burgers turbulence [1], the Kraichnan model of passive scalar advection and turbulence in kinematic magneto hydrodynamics 2] led to a better understanding of the limitations of phenomenological theories of turbulence. Also, it concentrated attention on more general concepts of turbulence, such as intermittency and breakdown of self-similarity.

In this paper we study breakdown of self-similarity in a model of diffusing, coagulating masses in the presence of a steady influx of monomers. Consider a $d$-dimensional hypercubic lattice whose sites are occupied by particles that carry positive masses. Multiple occupancy of a site is allowed. Given a configuration of particles, the system evolves in time as follows. At rate $D$, a particle hops to one of its $2 d$ nearest neighbor sites. At rate $2 \lambda$, two particles on the same site coalesce together to form a new particle whose mass is the sum of masses of its constituents. At rate $J / m_{0}$, a particle with mass $m_{0}$ is injected at a site. The parameter $J$ is the average mass flux into the system. For simplicity of analysis it is assumed that the rates $D$ and $\lambda$ do not depend on the particles masses. The initial condition is one in which there are no masses. We call this model the mass model (MM). A generalization of this model is one where the masses (or 'charges') could be positive or negative. Here, with rate $J_{c} / m_{0}^{2}$ particles of charge $m_{0}$ and $-m_{0}$ are input into the system. $J_{c}$ has the meaning of average influx of square of the charge. This model will be called the charge model $(\mathrm{CM})$. We will be interested in the continuous limit of these models.
A feature of the steady state of MM is the presence of constant flux of mass from small masses to large masses via coagulation. This is analogous to turbulent systems where there is a constant flux of energy from small wave numbers to large wave numbers via nonlinear interactions. Let $C_{n}\left(m_{1}, \ldots, m_{n}\right)(\Delta V)^{n} \prod_{i} d m_{i}$ be the probability of having particles of masses in the intervals $\left[m_{i}, m_{i}+d m_{i}\right]$ in a volume $\Delta V$. We ask how $C_{n}\left(m_{1}, \ldots, m_{n}\right)$ varies with mass when $m_{1}, \ldots, m_{n} \gg$ $m_{0}$. In particular what is the value of the homogeneity exponent $\gamma(n)$ defined through $C_{n}\left(\Gamma m_{1}, \ldots, \Gamma m_{n}\right)=$ $\Gamma^{-\gamma(n)} C_{n}\left(m_{1}, \ldots, m_{n}\right)$ ? The quantity in turbulence that is analogous to $C_{n}\left(m_{1}, \ldots, m_{n}\right)$ would be $\left\langle E_{k_{1}} \ldots E_{k_{n}}\right\rangle$, where $E_{k_{i}}$ is the energy corresponding to the wavenumber $k_{i}$.

MM has been studied in many different contexts. Examples include submonolayer epitaxial thin film growth by deposition of atoms onto a substrate in the limit when the distance between clusters is much larger than typical size of a cluster [3], river networks [4, 5], force fluctuation in in granular bead packs [6] and nonequilibrium phase transitions [7, 8]. It was one of the first models for self organized criticality wherein power laws are generated from simple dynamical rules. In this context, it also maps [9] onto the abelian directed sandpile model of self organized criticality [10]. Finally, the mean field limit of $\mathrm{MM}$ is mathematically similar to the kinetic equations of three-wave weak turbulence 11].

It was shown in Refs. 12, 13, 14 that in one dimension $\gamma(1)=4 / 3$ for $\mathrm{MM}$ and $\gamma(1)=5 / 3$ for CM. By studying the two point correlations, it was shown that in $d<2$, $\gamma(1)=(2 d+2) /(d+2)$ for MM and $\gamma(1)=(3 d+2) /(d+2)$ for CM 15, 16].

In this paper, using the renormalization group (RG) formalism, the exponent $\gamma(n)$ is calculated as an expansion in $\epsilon=2-d$ up to order $\epsilon$. In two dimensions, the upper critical dimension of the model, the logarithmic corrections to the mean field results for $C_{n}\left(m_{1}, \ldots, m_{n}\right)$ 
are calculated. Exact results are obtained for $C_{2}\left(m_{1}, m_{2}\right)$ in all dimensions. The Kolmogorov prediction for $\gamma(n)$ based on self similarity is shown to break down.

We first determine the dependence of $C_{n}\left(m_{1}, \ldots, m_{n}\right)$ on mass using a self-similarity conjecture similar to Kolmogorov's 1941 conjecture about the statistics of velocity increments in hydrodynamic turbulence. Assume that $C_{n}$ depends only on the masses $m_{i}$, mass flux $J$ and the diffusion coefficient $D$. The dimensions of the various parameters describing the continuous limit of the model are $[J]=M L^{-d} T^{-1},[D]=L^{2} T^{-1},\left[C_{n}\right]=L^{-n d} M^{-n}$ and $[m]=M$. There is a unique combination of $D$, $m$ and $J$ that has the dimension of $C_{n}$ given by $C_{n} \sim$ $\left(J D^{-1}\right)^{n d /(d+2)} m^{-\gamma_{k o l m}(n)}$, where

$$
\gamma_{k o l m}(n)=\left(\frac{2 d+2}{d+2}\right) n,
$$

is the Kolmogorov scaling exponent. As expected, the dependence of $\gamma_{\text {kolm }}$ on the index $n$ is linear, reflecting the assumed self-similarity of the statistics of the local mass distribution $N(\vec{x}, m, t)$. When $n=1, \gamma_{\text {kolm }}=$ $(2 d+2) /(d+2)$, which agrees with the result of an exact computation for $d<2$ [16].

The self-similarity conjecture assumes that $C_{n}$ does not depend on the following: the reaction rate $\lambda$, the lattice spacing, the position of the source $m_{0}$ and the box size $\Delta V d m_{1} \ldots d m_{n}$. The lack of dependence on the lattice spacing is expected due to the renormalizability of the effective field theory describing MM below two dimensions. We will however find an anomalous dependence of correlation functions on a length scale depending on the other parameters that leads to the violation of self-similarity.

Starting from the lattice model, it is possible to construct an effective field theory of MM using the formalism due to Doi and Zeldovich [17, 18. Furthermore, it is possible to establish an exact map between this field theory and the following stochastic integro-differential equation, [19, 20]:

$$
\begin{aligned}
& \left(\frac{\partial}{\partial t}-D \nabla^{2}\right) \phi(m)=\lambda \int_{0}^{m} d m^{\prime} \phi\left(m^{\prime}\right) \phi\left(m-m^{\prime}\right) \\
& -2 \lambda \phi(m) N+\frac{J}{m_{0}} \delta\left(m-m_{0}\right)+i \sqrt{2 \lambda} \phi(m) \eta(\vec{x}, t)(2)
\end{aligned}
$$

where $N=\int_{0}^{\infty} d m^{\prime} \phi\left(m^{\prime}\right), i=\sqrt{-1}$, and $\eta(\vec{x}, t)$ is white noise in space and time with $\left\langle\eta(\vec{x}, t) \eta\left(\vec{x}^{\prime}, t^{\prime}\right)\right\rangle=$ $\delta\left(t-t^{\prime}\right) \delta^{d}\left(\vec{x}-\vec{x}^{\prime}\right)$. All correlation functions of the mass distribution can be expressed in terms of the correlation functions of $\phi(m, \vec{x}, t)$ In particular,

$$
C_{n}(m) \approx \frac{1}{n !}\left\langle[\phi(m, \vec{x}, t)]^{n}\right\rangle,
$$

where $\langle\ldots\rangle$ denotes averaging with respect to noise $\eta$ 21].

Without the noise term, Eq. (2) reduces to the mean field Smoluchowski equation of the model. Thus, all the

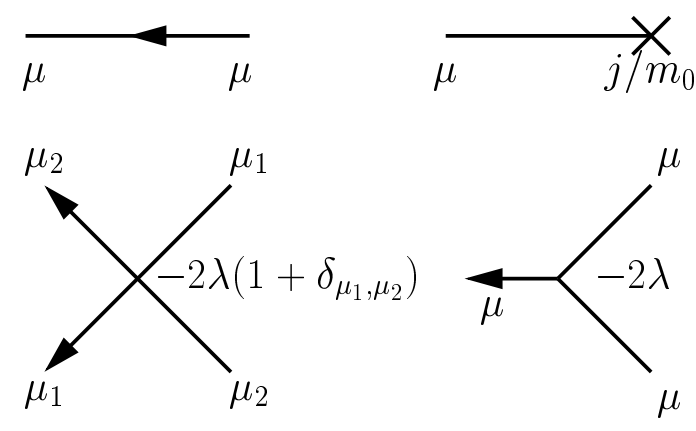

FIG. 1: Propagators and vertices of the theory.

fluctuation effects are encoded in the imaginary multiplicative noise term. Equation (2) simplifies after taking Laplace transform with respect to the mass variable 20]. Let $R_{\mu}(\vec{x}, t)=N(\vec{x}, t)-\int_{0}^{\infty} d m \phi(\vec{x}, m, t) e^{-\mu m}$. Then,

$$
\left(\frac{\partial}{\partial t}-D \nabla^{2}\right) R_{\mu}=-\lambda R_{\mu}^{2}+\frac{j}{m_{0}}+i \sqrt{2 \lambda} R_{\mu} \eta(\vec{x}, t),
$$

where $j=J\left(1-e^{-\mu m_{0}}\right)$, and the dependence of $R_{\mu}$ on $(\vec{x}, t)$ has been suppressed.

Equation (4) has the form of the stochastic rate equation of the $A+A \rightarrow A$ reaction in the presence of a source [22], reducing the the computation of the mean mass distribution in MM to solving a onespecies particle problem. However, in order to compute $C_{n}(m, t)$, the correlation functions of the form $\left\langle R_{\mu_{1}}(\vec{x}, t) R_{\mu_{2}}(\vec{x}, t) \ldots R_{\mu_{n}}(\vec{x}, t)\right\rangle$ need to be determined. These are non-trivial, as the stochastic fields $R_{\mu}(\vec{x}, t)$ 's are correlated for different values of $\mu$ via the common noise term in Eq. (44).

The set of Feynman rules for perturbative computation of correlation functions $C_{n}$ follows from Eq. (44) 23] and is summarized in Fig. [1] The $n$-point correlation function $\left\langle\prod_{i=1}^{n} R_{\mu_{i}}\left(\vec{x}_{i}, t_{i}\right)\right\rangle$ is given by the sum of all Feynman diagrams that have $n$ outgoing lines built out of blocks shown in Fig. 1]

Let $R_{m f}$, denoted by a thick line with a cross, be the sum of all tree diagrams with one outgoing line. The equation satisfied by $R_{m f}$ is shown in diagrammatic form in Fig. 22A: $d R_{m f} / d t=j / m_{0}-\lambda R_{m f}^{2}$. This corresponds to the noiseless limit of Eq. (4). Solving,

$$
R_{m f}(t)=\sqrt{\frac{j}{m_{0} \lambda}} \tanh \left(\sqrt{\frac{j \lambda}{m_{0}}} t\right) \stackrel{t \rightarrow \infty}{\longrightarrow} \sqrt{\frac{j}{m_{0} \lambda}} .
$$

To account for the noise term, we have to include diagrams with loops. To construct the loop expansion, it is convenient to introduce a tree level propagator $G_{m f}\left(x_{2} t_{2} ; x_{1} t_{1}\right)$. The equation obeyed by it is shown in Fig. 23. The solution is

$$
\frac{G_{m f}(\mathbf{2} ; \mathbf{1})}{G_{0}(\mathbf{2} ; \mathbf{1})}=\left[\frac{\cosh \sqrt{\frac{j \lambda}{m_{0}}} t_{1}}{\cosh \sqrt{\frac{j \lambda}{m_{0}}} t_{2}}\right]^{2} \stackrel{t_{1,2} \rightarrow \infty}{\longrightarrow} e^{-\Omega\left(t_{2}-t_{1}\right)}
$$


(A)

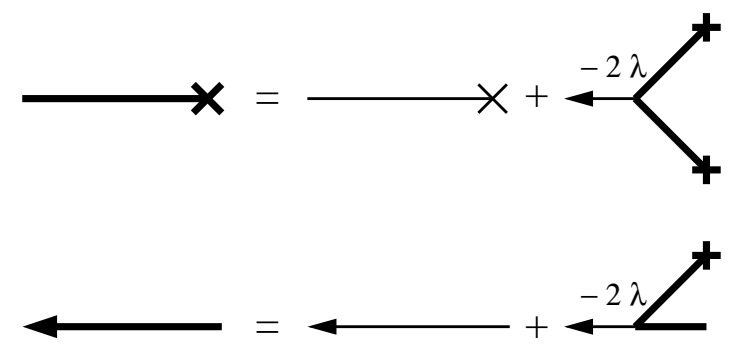

FIG. 2: Diagrammatic form of mean field equations for: (A) $R_{m f}$ and (B) $R R$-response function.

where $G_{0}$ is the Green's function of the linear diffusion equation, $\mathbf{2}=\left(\vec{x}_{2}, t_{2}\right), \mathbf{1}=\left(\vec{x}_{1}, t_{1}\right)$, and $\Omega=2 \sqrt{j \lambda / m_{0}}$ is the inverse of the mean field response time. All terms in the loop expansion constructed using the vertices of Fig $1 . G_{m f}$ and $R_{m f}$ are finite in $d<2$.

We now show that the loop expansion corresponds to weak coupling expansion with respect to $\lambda$. Consider a diagram contributing to $\left\langle R_{\mu}^{n}\right\rangle$ that has $L$ loops, $V$ vertices and $N R_{m f}$-lines. The $\lambda$ factors arise from $L d$ momentum integrals $\left(\lambda^{L d / 4}\right), V$ time integrals $\left(\lambda^{-V / 2}\right), N R_{m f}$ lines $\left(\lambda^{-N / 2}\right)$ and $V$ vertices $\left(\lambda^{V}\right)$. Thus, the corresponding Feynman integral is proportional to $\lambda^{-N / 2+V / 2+L d / 4}$. Also, the number of triangular vertices in the graph equals $N-n$ and the number of quartic vertices is equal to the number of loops $L$, i.e., $V=L+N-n$. Thus, any $L$-loop graph contributing to the mean mass distribution is proportional to $\lambda^{-\frac{n}{2}+L\left(1+\frac{d+2}{4}\right)}$. We conclude that loop expansion corresponds to the perturbative expansion of $\left\langle R_{\mu}^{n}\right\rangle$ around $R_{m f}^{n}$ with the parameter $\lambda^{\frac{2+d}{4}}$.

The conditions under which the loop corrections to the tree level answer can be neglected may be derived using dimensional analysis. The scale of diffusive fluctuations is given by the only constant of dimension length which can be constructed out of $D$ and and $j / m_{0}: L_{D}=$ $\left(m_{0} D / j\right)^{1 /(d+2)}$. The dimensionless expansion parameter in the loop expansion above is therefore $g\left(L_{D}\right)=\lambda L_{D}^{\epsilon}$, where $\epsilon=2-d$. The large mass behavior of $C_{n}(m)$ is determined by the small- $\mu$ behavior of $\left\langle R_{\mu}^{n}\right\rangle$. In $d<2$, $g_{0} \rightarrow \infty$ when $\mu \rightarrow 0$ and the loop expansion breaks down. Thus, a re-summation of loop expansion is needed to extract the $\mu \rightarrow 0$ behavior of $R_{\mu}$. This will be done using dynamical $\mathrm{RG}$ formalism.

We first examine $\left\langle R_{\mu}\right\rangle$. It was shown in Ref. [19, 22] that renormalization of the coupling constant alone regularizes the loop expansion for $\left\langle R_{\mu}\right\rangle$ when $\epsilon \rightarrow 0$. In particular, $\left\langle R_{\mu}\right\rangle$ has no anomalous dimension and therefore has the form $L_{D}^{-d} f\left[g_{R}, L_{0} / L_{D}\right]$ where $L_{0}$ is a reference scale. $g_{R}$ is the renormalized reaction rate and is related to $g\left(L_{0}\right)$ by

$$
g_{R}=\frac{g\left(L_{0}\right)}{1+g\left(L_{0}\right) / g^{*}},
$$

where $g^{*}=(8 \pi)^{d / 2}[2 \Gamma(\epsilon / 2)]^{-1}[19,24] .\left\langle R_{\mu}\right\rangle$ does not

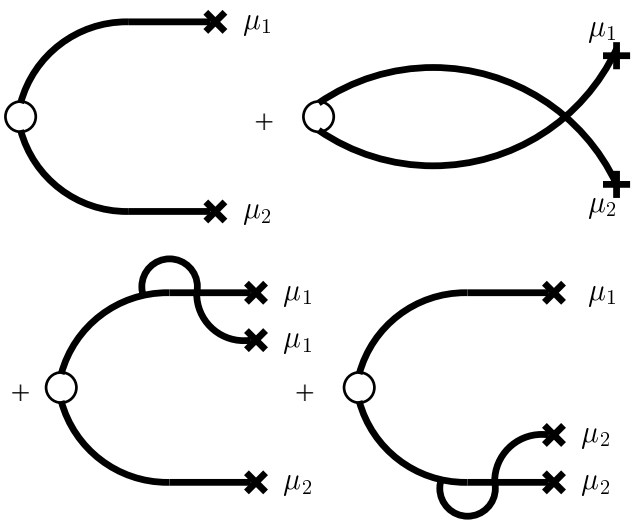

FIG. 3: Zero and one loop Feynman diagrams contributing to $\left\langle R_{\mu_{1}}(\vec{x}, t) R_{\mu_{2}}(\vec{x}, t)\right\rangle$. The circles mean that all outgoing lines terminate at the same spatial point.

depend on the length scale $L_{0}$. Under RG, $g_{R}$ goes to $g^{*}$ as $L_{D} \rightarrow \infty$ [22]. Hence, $\left\langle R_{\mu}\right\rangle \sim L_{D}^{-d}$. Large $L_{D}$ corresponds to small $\mu$. Then $j / m_{0} \approx J \mu$, and $R_{\mu} \sim$ $(J \mu)^{d /(d+2)}$. The inverse Laplace transform gives

$$
C_{1}(m) \sim \frac{\left(D^{-1} J\right)^{d /(d+2)}}{m^{\gamma(1)}}, \quad d<2,
$$

where $\gamma(1)=(2 d+2) /(d+2)$. This result is exact to all orders in $\epsilon$.

The RG analysis shows that $C_{1}$ depends only on $J, m$ and $D$ and thus verifies the self similarity conjecture for $n=1$. Therefore, it is not surprising that $\gamma(1)$ coincides with $\gamma_{\text {kolm }}(1)$.

We now examine higher order correlation functions of the form $\left\langle R_{\mu_{1}}(\vec{x}, t) R_{\mu_{2}}(\vec{x}, t) \ldots\right\rangle$. Now, extra ultraviolet divergences, that do not get canceled by coupling constant renormalization, appear in the perturbative expansion of these composite operators 25]. These extra divergences lead to a spectrum of anomalous dimensions which are calculated below.

The diagrams contributing to $\left\langle R \mu_{1} R \mu_{2}\right\rangle$ up to one loop are shown in Fig. 3. It is straightforward to generalize them to $n>2$. For $n \geq 2$, diagrams contain $n$ outgoing lines and $\left(\begin{array}{l}n \\ 2\end{array}\right)$ connected one loop diagrams. Computing the diagrams using equations (5), (6) and simplifying, we obtain

$$
\left\langle\prod_{i=1}^{n} R_{\mu_{i}}\right\rangle=\prod_{i=1}^{n}\left\langle R_{\mu_{i}}\right\rangle\left[1-\frac{n(n-1) \lambda \Gamma(\epsilon / 2)}{(8 \pi D)^{d / 2}\left(\Omega_{1}+\Omega_{2}\right)^{\epsilon / 2}}+\ldots\right] .
$$

Expressed in terms of the renormalized coupling $g_{R}$, Eq. (9) reduces to

$$
\left\langle\prod_{i=1}^{n} R_{\mu_{i}}\right\rangle=\prod_{i=1}^{n}\left\langle R_{\mu_{i}}\left(g_{R}\right)\right\rangle\left[1-\frac{n(n-1) g_{R}}{4 \pi \epsilon}+O\left(g_{R}^{2}\right)\right] .
$$

Since coupling constant renormalization removes all singularities in $\left\langle R_{\mu}\left(g_{R}\right)\right\rangle$, there are no singularities in the product $\prod_{i=1}^{n}\left\langle R_{\mu_{i}}\left(g_{R}\right)\right\rangle$ when $\epsilon \rightarrow 0$. 
The remaining singularity is canceled by multiplicative renormalization of the composite operators. Let $\left\langle\prod_{i=1}^{n} R_{\mu_{i}}\right\rangle_{R}=Z_{n}\left\langle\prod_{i=1}^{n} R_{\mu_{i}}\right\rangle$, where $Z_{n}$ is fixed by the condition that $\left\langle\prod_{i=1}^{n} R_{\mu_{i}}\right\rangle_{R}$ is not singular in $\epsilon$ [26]. Thus $Z_{n}=1+n(n-1) g_{R} /(4 \pi \epsilon)+O\left(\epsilon^{2}\right)$. The bare $\left\langle\prod_{i=1}^{n} R_{\mu_{i}}\right\rangle$ is independent of the reference scale $L_{0}$. Thus,

$$
L_{0} \frac{\partial}{\partial L_{0}}\left[Z_{n}^{-1}\left\langle\prod_{i=1}^{n} R_{\mu_{i}}\right\rangle_{R}\right]=0 .
$$

Let $L_{i}=\left[m_{0} D / j\left(\mu_{i}\right)\right]^{1 /(d+2)}$. By dimensional analysis, $\left\langle\prod_{i=1}^{n} R_{\mu_{i}}\right\rangle=L_{0}^{-2 d} f\left(L_{i} / L_{0}, g_{R}\right)$, where $f$ is a dimensionless scaling function. Substituting into Eq. (11), and solving the resulting equation in the limit $L_{i} \gg L_{0}$, we obtain $\left\langle\prod_{i=1}^{n} R_{\mu_{i}}\right\rangle_{R} \sim \Phi_{1} \prod_{i=1}^{n} L_{i}^{-d-\epsilon(n-1) / 2}$, where $\Phi_{1}$ is a scaling function of the variables $L_{i} / L_{j}$. An inverse Laplace transform gives

$$
C_{n}\left(m_{1}, \ldots, m_{n}\right) \sim \Phi_{2} \prod_{i=1}^{n} \frac{1}{m_{i}}\left(\frac{J}{D m_{i}}\right)^{\frac{d+\epsilon(n-1) / 2}{d+2}}
$$

$\Phi_{2}$ is a scaling function of the variables $m_{i} / m_{j}$ and the parameters $\Delta V, m_{0}$ and $\lambda$. In the limit when $m_{0} \rightarrow 0$ and $\lambda \rightarrow \infty$, we expect the $\Delta V$ dependence of $\Phi_{2}$ to be $\Phi_{2} \sim(\Delta V)^{\frac{\epsilon n(n-1)}{2 d}}$. We then conclude that

$$
\gamma(n)=\left(\frac{2 d+2}{d+2}\right) n+\left(\frac{\epsilon}{d+2}\right) \frac{n(n-1)}{2}+O\left(\epsilon^{2}\right) .
$$

The first term coincides with $\gamma_{\text {kolm }}$ [see Eq. (1)]. The presence of the second term leads to breakdown of self similarity. This is due to the effective anticorrelation between the particles. The self similarity conjecture for MM is equivalent to a renormalized mean field theory in which only the coupling renormalization is taken into account. Therefore, it cannot take into account correlations between particles.

Though, Eq. (13) is an $\epsilon$-expansion, it is still possible to confirm that $\gamma(n) \neq \gamma_{\text {kolm }}(n)$ in $d<2$ by computing $\gamma(2)$ exactly. From the definition of $\gamma(2)$, it follows that $\left\langle R_{\mu_{1}} R_{\mu_{2}}\right\rangle=\left(\mu_{1} \mu_{2}\right)^{\gamma(2) / 2-1} \psi\left(\mu_{1} / \mu_{2}\right)$, where $\psi(x)$ is an unknown scaling function with the property $\psi(x)=\psi(1 / x)$. We need to know the $\mu_{1}, \mu_{2} \rightarrow 0$ behavior of $\left\langle R_{\mu_{1}} R_{\mu_{2}}\right\rangle$. Averaging Eq. (4) with respect to noise and setting $\partial_{t}\left\langle R_{\mu}\right\rangle=0$ in the large time limit, we find that $\left\langle R_{\mu} R_{\mu}\right\rangle=j /\left(\lambda m_{0}\right) \approx J \mu / \lambda$ for $\mu \ll m_{0}^{-1}$. Comparing this result with the above scaling form, we find that $\gamma(2)=3$ exactly, which coincides with Eq. (13) with terms of $O\left(\epsilon^{2}\right)$ and higher order set to zero.

Note that $\gamma(2)$ does not depend on the dimension $d$. The result $\gamma(2)=3$ is a counterpart of the $4 / 5$-th law of Navier-Stokes turbulence and is due to conservation of mass flux. Recall that the $4 / 5$ law states that, in the inertial range the third order structure point function of velocity field scales as the first power of separation. While the Kolmogorov theory respects 4/5-law in NavierStokes turbulence, it violates $\gamma(2)=3$ law in MM.

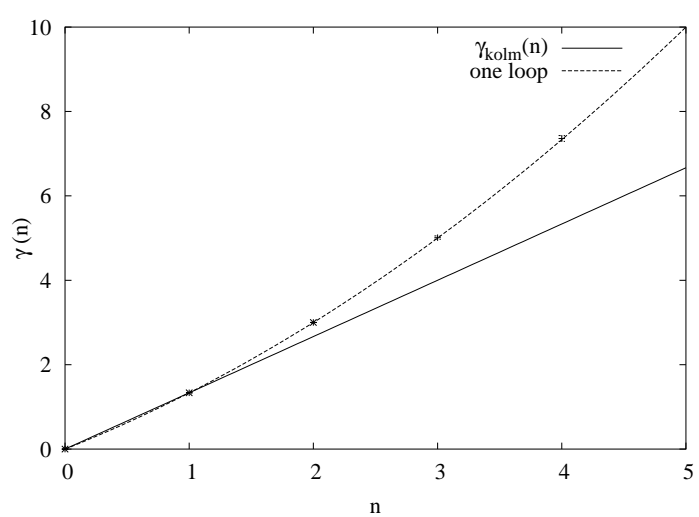

FIG. 4: The variation of homogeneity exponent $\gamma(n)$ with $n$ is shown for one dimension. The dotted line corresponds to Eq. (13) with $\epsilon=1$ and terms of order $\epsilon^{2}$ and higher set to zero. The values of $\gamma(0), \gamma(1)$ and $\gamma(2)$ are exact while $\gamma(3)$ and $\gamma(4)$ were obtained by Monte Carlo simulations. The simulations were performed on a lattice of size $10^{5}$ and averaged $2 \times 10^{7}$ times with $J=4 D$.

The terms $O\left(\epsilon^{2}\right)$ and higher in Eq. (13) are either very small or equal to zero when $d=1$. We showed their absence for $n=1,2$ by means of an exact computation. For $n=3,4$ we verify their smallness in one dimensions

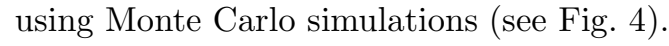

In two dimensions, logarithmic corrections to the Kolmogorov scaling are expected. These can be calculated exactly using the RG method. We present the final results here:

$$
C_{n}(m) \sim \frac{J^{n / 2}[\ln (m)]^{n-n^{2} / 2}}{m^{3 n / 2}}\left[1+O\left(\frac{1}{\ln (m)}\right)\right], d=2 .
$$

The logarithmic corrections vanish for $n=2$. This is consistent with the exact result for $C_{2}(m) \sim m^{-3}$, which is valid in all dimensions.

It is straightforward to extend the above results to CM. Kolmogorov theory for the charge model is developed using the flux of charge squared $J_{c}$ as a self-similarity parameter. This leads to Kolmogorov spectrum for CM, $\gamma_{\text {kolm }}^{C M}=\left(\frac{3 d+2}{d+2}\right) n$. The stochastic equation for $\mathrm{CM}$ is the same as Eq. (41) modulo a redefinition of the source term: $\left.j / m_{0} \rightarrow 2 J_{c}\left(1-\cosh \left(\mu m_{0}\right)\right) / m_{0}^{2}\right)$. Therefore the RG analysis presented for MM goes through with just one modification: the scale of diffusive fluctuations in the charge model is $L_{D}=\left(J_{c} D^{-1} \mu^{-2}\right)^{\frac{1}{d+2}}$ for $\mu \rightarrow 0$. The result of the analysis is

$$
\gamma^{C M}(n)=\gamma_{\text {kolm }}^{C M}(n)+\left(\frac{\epsilon}{d+2}\right) n(n-1)+O\left(\epsilon^{2}\right) .
$$

In complete analogy with MM case, $O\left(\epsilon^{2}\right)$ terms in the above answer vanish for $n=1$ and $n=2$. Kolmogorov theory correctly predicts the scaling of average charge distribution in CM, but fails to predict the scaling of 
multi point probability distributions of charge. Relation $\gamma^{C M}(2)=4$ reflects conservation of flux of charge squared and is satisfied in all dimensions.

To summarize, we considered two models of aggregation with input which had features qualitatively similar to turbulence. For these models, we were able to compute the statistics of the local mass (charge) distribution and compare the results against the predictions from Kolmogorov theory. It turned out that Kolmogorov selfsimilarity conjecture is equivalent to a renormalized mean field theory approximation which correctly accounts for effects of reaction rate renormalization in both $\mathrm{MM}$ and CM. As a result, the answers for average mass (charge) distribution derived from Kolmogorov theory turned out to be correct. However, Kolmogorov theory failed to predict multi point distributions of mass (charge) correctly: small scale correlations between diffusing, coagulating particles are relevant for the multi point statistics, but cannot be accounted for by coupling constant renormalization alone.

We would like to thank G. Falkovich for useful discussions. RR acknowledges support from NSF grant DMR0207106. CC acknowledges support from Marie Curie grant HPMF-CT-2002-02004. OZ thanks the hospitality at Brandeis University, where part of this work was done.

[1] U. Frisch, Turbulence: The Legacy of A. N. Kolmogorov (Cambridge University Press, Cambridge, 1995).

[2] G. Falkovich, K. Gawȩdzki, and M. Vergassola, Rev. Mod. Phys. 73, 913 (2001).

[3] P. L. Krapivsky, J. F. F. Mendes, and S. Redner, Phys. Rev. B 59, 15950 (1999).

[4] A. E. Scheidegger, Bull. I. A. S. H. 12, 15 (1967).

[5] P. S. Dodds and D. H. Rothman, Phys. Rev. E 59, 4865
(1999).

[6] S. N. Coppersmith, C. h Liu, S. Majumdar, O. Narayan, and T. A. Witten, Phys. Rev. E 53, 4673 (1996).

[7] S. N. Majumdar, S. Krishnamurthy, and M. Barma, Phys. Rev. E 61, 6337 (2000).

[8] R. Rajesh, Phys. Rev. E 69, 036128 (2004).

[9] D. Dhar, preprint arXiv:cond-mat/9909009 (1999).

[10] D. Dhar and R. Ramaswamy, Phys. Rev. Lett. 63, 1659 (1989).

[11] V. Zakharov, V. Lvov, and G. Falkovich, Kolmogorov Spectra of Turbulence (Springer-Verlag, Berlin, 1992).

[12] H. Takayasu, Phys. Rev. Lett. 63, 2563 (1989).

[13] V. Privman, ed., Nonequilibrium Statistical Mechanics in one dimensions (Cambridge University Press, Cambridge, 1997), pp. 181-201.

[14] G. Huber, Physica A 170, 463 (1991).

[15] S. N. Majumdar and C. Sire, Phys. Rev. Lett. 71, 3729 (1993).

[16] R. Rajesh and S. N. Majumdar, Phys. Rev. E 62, 3186 (2000).

[17] M. Doi, J. Phys. A 9, 1465 (1976).

[18] Y. B. Zel'dovich and A. A. Ovchinnikov, Soviet Phys. JETP 47, 829 (1978).

[19] B. P. Lee, J. Phys. A 27, 2633 (1994).

[20] O. Zaboronski, Phys. Lett. A 281, 119 (2001).

[21] C. Connaughton, R. Rajesh, and O. Zaboronski, unpublished.

[22] M. Droz and L. Sasvári, Phys. Rev. E 48, R2343 (1993).

[23] C. Itzykson and J. M. Drouffe, Statistical Field Theory, Cambridge monographs on mathematical physics (Cambridge University Press, Cambridge, 1994).

[24] L. Peliti, J. Phys. A 19, L365 (1986).

[25] J. Zinn-Justin, Quantum Field Theory and Critical Phenomena (Oxford University Press, Oxford, U.K, 2002).

[26] The problem of renormalization of composite operators is simple for MM because operators of lower order do not contribute to the renormalization of operators of higher order. The absence of mixing is due to the absence of vertices increasing the number of outgoing lines in any Feynman diagram 